Sharif University of Technology
Scientia Iranica
SCIENTIA
I RAN I CA

\title{
Reliability analysis of foundation settlement by stochastic response surface and random finite-element method
}

\author{
A. Johari* and A. Sabzi \\ Department of Civil and Environmental Engineering, Shiraz University of Technology, Shiraz, Iran.
}

Received 21 October 2015; received in revised form 20 April 2016; accepted 22 August 2016

\author{
KEYWORDS \\ Foundation \\ settlement; \\ Spatial variability; \\ Random fields; \\ Stochastic response \\ surface method; \\ Random finite- \\ element method; \\ Karhunen-Loeve \\ expansion.
}

\begin{abstract}
This paper presents a reliability-based analysis of strip-footing settlement by Stochastic Finite-Element Method (SFEM). The Stochastic Response Surface Method (SRSM) and Random Finite-Element Method (RFEM) are used as two formulations of SFEM. The elastic properties of soil are considered as spatial random variables and modeled as cross correlated log-normal random fields. Random field discretization is done by Karhunen-Loeve (K-L) expansion. Two programs were coded by MATLAB so as to take full advantages of its matrix operations, and in an illustrative example, it was shown that the results of SRSM are close to RFEM; however, the consumed time in RFEM is at most 50 times longer than that in SRSM. Using the faster method, SRSM, it is concluded that considering the spatial variability of soil parameters in stochastic analysis is necessary.
\end{abstract}

(C) 2017 Sharif University of Technology. All rights reserved.

\section{Introduction}

The soil properties are inherently uncertain parameters. These properties are spatial variables and vary from one point in space to another. This leads to the necessity of representing the soil parameters as characterized random fields. In conventional approaches to foundation settlement determination, average values of soil parameters are considered and parameters' uncertainties are taken into account by applying a global Factor of Safety (FS). Probabilistic analysis provides a tool to consider the uncertain parameters in analysis.

There have been some scientific efforts directed at applying reliability analysis to the civil engineering tasks $[1,2]$.

Many methods have been developed for prob-

\footnotetext{
*. Corresponding author.

E-mail address: johari@sutech.ac.ir (A. Johari)
}

abilistic/stochastic analysis of foundations on soils. Some of these are Monte Carlo Simulation (MCS), First-Order Reliability Method (FORM), SecondOrder Reliability Method (SORM), Perturbation theory, SRSM, etc. [3]. For example, Shyamala and Dodagoudar [4] used Point Estimation Method (PEM) and First-Order Second Moment (FOSM) along with the non-linear finite-element analysis to evaluate the reliability index in reliability analysis of shallow foundation settlements. The mentioned methods are clubbed with finite-element method to stochastic analysis of foundation settlement giving rise to methods like SFEM and RFEM. Among above methods, the RFEM and SRSM are two powerful methods to propagate input parameters uncertainties in finite-element models.

In RFEM, the random fields are combined with the finite-element method through MCS. The RFEM has been widely used in geotechnical problems [5-9]. For example, Fenton and Griffiths [6] presented reliability analysis of foundation settlement by modeling the soil as spatially random media. Prediction of 
settlement below the foundation was obtained using the finite-element method. Griffiths and Fenton [7] compared the reliability results of foundation settlement, obtained by stochastic finite-element method based on first-order second-moment approximations, with the result of random finite-element method based on generation of random fields combined with Monte Carlo simulations. Pieczynska-Kozlowska et al. [10] studied the influence of embedment, soil self-weight, and anisotropy of random field on bearing capacity of foundation using RFEM.

In SRSM, the complex numerical model is replaced by an analytical one, which is less timeconsuming compared with original numerical model (deterministic model). The SRSM can be adopted in both linear and nonlinear problems, and all statistical moments of outputs can be computed using this method. The SRSM has been used in many problems [11-19]. Among important contributions are the research studies of Huang et al. [14], Li et al. [17], Li and Zhang [18], and Jiang et al. [16]. Huang et al. [14] presented an extended stochastic response-surface method for problems in which physical properties exhibit spatial random variation by using collocation points as samples for constructing the output response surface. Li et al. [17] proposed a stochastic response surface method for reliability analysis involving correlated non-normal random variables. They formulated the closed-form expressions for fourth to sixth order Hermit polynomial chaos expansions involving any number of random variables. $\mathrm{Li}$ and Zhang [18] explored a method for uncertainty analysis of flow in random porous media by combining the KL expansion and the SRSM based on Probabilistic Collocation Method (PCM). Jiang et al. [16] proposed a non-intrusive stochastic finite-element method for slope reliability analysis considering spatial variability of shear strength parameters. They adopted the K-L expansion to discretize the 2-Dimentional (2-D) crosscorrelated non-Gaussian random fields of spatially variable shear strength parameters.

The objective of this paper is to analyze the reliability of strip foundation settlement by SRSM, which requires low computational time and low memory requirements; then, a comparison will be made between the obtained results with those of RFEM. In reliability analysis by SRSM, the model outputs are represented as functions of standard normal variables by polynomial chaos expansion. The unknown coefficients in the polynomial chaos expansion are determined using a probabilistic collocation method. For modeling the soil behavior, elastic-perfectly plastic Mohr-Coulomb yield criterion, which is common in geotechnical problems, is used. In elastic-perfectly plastic models, hardening or softening which shows a more real behavior of soil cannot be considered. Each spatially random variable is modeled as cross correlated log-normal 2-D random field. The log-normal distribution is selected by the fact that the values of soil parameters are strictly positive. The well-known K-L expansion is used for random filed discretization. Furthermore, an example is presented to compare the predictions of the SRSM and RFEM on the reliability analysis of settlement strip foundation.

\section{Shallow foundation settlement}

Foundation settlement will occur when a foundation is subjected to load. Excessive settlements may lead to serviceability problems and the desired use of the structure may be impaired. The foundation settlement depends on soil type and the water table level. It consists of three components: immediate settlement, consolidation settlement, and secondary compression. Classical formulations are available for computing these components of settlements. Numerical methods, such as finite-element and finite-difference methods, can be used to calculate foundation settlement. These methods provide the advantage of idealizing the material behavior of soil, which is non-linear with plastic deformations and path dependent, in a more rational manner. In this study, the finite-element method is used to determine the foundation settlement.

\section{Random field modeling of soil properties}

The soil properties are spatial variables and vary from one point in space to another. The spatial variability of soil properties can be due to variations in mineralogical composition, conditions during deposition, stress history, and physical and mechanical decomposition processes [20,21]. This leads to the necessity of representing the soil parameters as characterized random fields. Spatial variability of soil properties can be modeled using theory of random fields [22]. In the theory of random fields, at any location within a soil layer, the soil parameter is an uncertain quantity or a random variable which is characterized by a probability distribution and is correlated with the random variables at adjacent locations [20].

The values of a soil parameter are correlated at different points of a field. The spatial correlation of soil parameter is considered by autocorrelation function. The autocorrelation function of a given soil parameter can be estimated from the measured data of the parameter at different locations [23]. In this study, for all uncertain parameters, a squared exponential 2$\mathrm{D}$ autocorrelation function is assumed with different autocorrelation distances in the horizontal and vertical directions as follows:

$$
\rho\left(\mathbf{x}, \mathbf{x}^{\prime}\right)=\exp \left(-\frac{\left|x-x^{\prime}\right|}{l_{x}}-\frac{\left|y-y^{\prime}\right|}{l_{y}}\right),
$$


where $\mathbf{x}$ and $\mathbf{x}^{\prime}$ are spatial coordinates, and $l_{x}$ and $l_{y}$ are autocorrelation lengths in horizontal and vertical directions, respectively. Small values of the autocorrelation lengths imply a rapid fluctuation about the soil parameter. However, high values imply a slowly varying soil parameter. In the case of $2-\mathrm{D}$ problems, it is assumed that out-of-plane autocorrelation length is infinite. If spatial variability of out-of-plane uncertain parameter be considered, 3-dimensional finiteelement analysis should be done to consider its effects.

\subsection{Discretization of random fields}

The numerical methods, such as finite-element formulation, have discrete nature; therefore, a continuousparameter random field must also be discretized into random variables. This process is commonly known as a discretization of a random field. There are several methods to discretize a random field in the literature [24]. More efficient approaches for discretization of random fields are series expansion methods.

The series expansion methods can be done by three methods [24]: the K-L expansion, Orthogonal Series Expansion (OSE), and the Expansion Optimal Linear Estimation (EOLE) methods. K-L expansion is able to use a few terms to capture the characteristic of the strongly correlated random fields [25]. In this study, the K-L expansion is used. This method is introduced briefly in the next subsection.

\subsubsection{Karhunen-Loève expansion}

The K-L expansion of a random field is based on the spectral decomposition of its autocorrelation function $\rho\left(\mathbf{x}, \mathbf{x}^{\prime}\right)$. The set of deterministic functions, over which any realization of field $H(\mathbf{x}, \theta)$ is expanded, is defined by eigenvalue problem:

$$
\int_{\Omega} \rho\left(\mathbf{x}, \mathbf{x}^{\prime}\right) \varphi_{i}\left(\mathbf{x}^{\prime}\right) d \Omega_{x^{\prime}}=\lambda_{i} \varphi_{i}(\mathbf{x})
$$

where $\mathbf{x}$ and $\mathbf{x}^{\prime}$ denote the coordinates of two points in space; $\varphi_{i}(\mathbf{x})$ and $\lambda_{i}$ are eigenfunctions and eigenvalues of one-dimensional autocorrelation function $\rho\left(\mathbf{x}, \mathbf{x}^{\prime}\right)$, respectively. The eigenmodes of the separable multidimensional autocorrelation function are calculated through multiplying them by the eigenmodes obtained from Eq. (2). This integral can be solved analytically or numerically $[26,27]$. In this study, the closed form presented by Zhang and Lu [28] is used. The series expansion of a random field $H(\mathbf{x}, \theta)$ is expressed as follows:

$$
H(\mathbf{x}, \theta)=\mu+\sum_{i=1}^{\infty} \sigma \sqrt{\lambda_{i}} \varphi_{i}(\mathbf{x}) \xi_{i}(\theta), \quad \mathbf{x} \in \Omega,
$$

where $\mu$ is mean and $\sigma$ is standard deviation of the field; $\xi_{i}(\theta)$ is a vector of uncorrelated standard normal variables, and $\theta$ indicates the random nature of the $H(\mathbf{x}, \theta)$. Practically, the series is approximated by a finite number of terms:

$$
H(\mathbf{x}, \theta)=\mu+\sum_{i=1}^{M} \sigma \sqrt{\lambda_{i}} \varphi_{i}(\mathbf{x}) \xi_{i}(\theta), \quad \mathbf{x} \in \Omega .
$$

The value of $M$ strongly depends on the desired accuracy and on the autocorrelation function of the random field [29]. Small values of the autocorrelation lengths will lead to a significant increase in the number of the K-L terms $(M)$.

\subsubsection{Cross correlated lognormal random fields}

Typically, more than one random soil parameter, such as Young's modulus, Poisson's ratio, the cohesion, and the friction angle, is involved in geotechnical problems. Among these, some soil parameters show the degree of dependency on each other. In other words, these parameters are cross correlated. Cross correlation structure between each pair of simulated fields was simply defined by a cross correlation coefficient $[29,30]$. Cross correlated Gaussian random field is expressed as follows:

$$
\begin{aligned}
& H_{i}(\mathbf{x}, \theta)=\mu_{i}+\sum_{j=1}^{M} \sigma_{i} \sqrt{\lambda_{j}} \varphi_{j}(\mathbf{x}) \chi_{i, j}(\theta), \\
& (\text { for } i=E, v),
\end{aligned}
$$

where $\chi_{i, j}$ is the correlated random vector whose $k$ th column, $\chi_{k}$, is given by [16]:

$$
\chi^{k}=\left[\chi_{E}^{k}, \chi_{v}^{k}\right]=\left[\xi_{E}^{k}, \xi_{E}^{k} \cdot R_{E, v}+\xi_{v}^{k} \sqrt{1-R_{E, v}^{2}}\right],
$$

where $\xi_{i}(i=E$ or $v)$ is the independent standard normal variables which correspond to the random variables used to discretize the random fields using the K-L expansion in Eq. (4), and $R_{E, v}$ is cross correlation coefficient among $E$ and $v$.

As the soil parameters are always positive, the Gaussian random field is not applicable. In this study, the variables are considered as lognormal random fields. Cross correlated lognormal random fields can be obtained by exponentiation of the approximate cross correlated Gaussian random fields from Eq. (5) as follows $[11,16,29]$ :

$$
H_{i}(\mathbf{x}, \theta)=\exp \left(\mu_{\ln i}+\sum_{j=1}^{M} \sigma_{\ln i} \sqrt{\lambda_{j}} \varphi_{j}(\mathbf{x}) \chi_{i, j}(\theta)\right)
$$

(for or $i=E, v)$

where $\mu_{\ln i}$ and $\sigma_{\ln i}$ are the mean and standard deviation of the Gaussian random variable $\ln i$, respectively: 


$$
\mu_{\ln i}=\ln \mu_{i}-\sigma_{\ln i}^{2} / 2 \text { and } \sigma_{\ln i}=\sqrt{\ln \left(1+\left(\sigma_{i} / \mu_{i}\right)^{2}\right)}
$$

\section{Stochastic response surface method}

The stochastic response surface method can be viewed as a conceptual extension of the traditional Response Surface Method (RSM) [15]. In this method, the complex numerical model (or original numerical model) is replaced by an analytical model called surrogate model or meta-model. The Probability Density Function (PDF) of system response can be computed easily by applying MCS on the meta-model, which is less timeconsuming in comparison to original numerical model. The major advantage of SRSM is that it allows the existing deterministic numerical codes, such as a finiteelement analysis code, to be used as a black-box within the method. The steps of SRSM can be written as follows [11,13-15]:

1. Representation of the stochastic input parameters in terms of independent Standard Random Variables (SRVs). In this study, this task is done using K-L expansion (Eq. (4)).

2. An output of a model open to influence by any number of model inputs. Hence, any general functional representation of uncertainty in model outputs should take into account uncertainties in all inputs. For this purpose, the output parameter must be represented as functions of the same set of SRVs, used for representation of the stochastic input parameters. This task is done using Hermit polynomial chaos expansion as follows:

$$
\begin{aligned}
y(\theta)= & a_{0} \Gamma_{0}+\sum_{i_{1}=1}^{N} a_{i_{1}} \Gamma_{1}\left(\xi_{i_{1}}\right) \\
& +\sum_{i_{1}=1}^{N} \sum_{i_{2}=1}^{i_{1}} a_{i_{1} i_{2}} \Gamma_{2}\left(\xi_{i_{1}}(\theta), \xi_{i_{2}}(\theta)\right) \\
& +\sum_{i_{1}=1}^{\infty} \sum_{i_{2}=1}^{i_{1}} \sum_{i_{3}=1}^{i_{2}} a_{i_{1} i_{2} i_{3}} \Gamma_{3}\left(\xi_{i_{1}}(\theta), \xi_{i_{2}}(\theta) \cdot \xi_{i_{3}}(\theta)\right) \\
& +\ldots+\sum_{i_{1}=1}^{\infty} \sum_{i_{2}=1}^{i_{1}} \sum_{i_{3}=1}^{i_{2}} \ldots \sum_{i_{N}=1}^{N} a_{i_{1} i_{2} \ldots i_{N}} \\
& \Gamma_{N}\left(\xi_{i_{1}}(\theta), \xi_{i_{2}}(\theta), \ldots, \xi_{i_{N}}(\theta)\right)
\end{aligned}
$$

where $y$ is model output; $a_{i_{0}}, a_{i_{1}}, a_{i_{1} i_{2}}, a_{i_{1} i_{2} i_{3}}, \ldots$ are the coefficients to be estimated, in which $N$ is the number of SRVs used to represent stochastic input parameters; $\xi=\left(\xi_{i_{1}}, \xi_{i_{2}}, \ldots, \xi_{i_{N}}\right)$ is a vector of independent standard normal variables, and $\Gamma_{N}\left(\xi_{i_{1}}, \xi_{i_{2}}, \ldots, \xi_{i_{N}}\right)$ are the multi-dimensional Hermit polynomials of degree $p$ given by:

$$
\Gamma_{p}\left(\xi_{i_{1}}, \ldots, \xi_{i_{N}}\right)=(-1)^{p} e^{\frac{1}{2} \xi^{T} \xi} \frac{\partial^{N}}{\partial \xi_{i_{1}} \ldots \partial \xi_{i_{N}}} e^{-\frac{1}{2} \xi^{T} \xi}
$$

For notational simplicity, Eq. (7) is rewritten as follows:

$$
y(\theta)=\sum_{j=0}^{P-1} c_{j} \psi_{j}(\xi(\theta)),
$$

where there is a correspondence between $\Gamma_{N}$ $\left(\xi_{i_{1}}, \xi_{i_{2}}, \ldots, \xi_{i_{N}}\right)$ and $\psi_{j}(\xi(\theta))$ and also between their corresponding coefficients. $P$ is the number of unknown coefficients calculated by:

$$
P=\frac{(N+p) !}{N ! p !}
$$

Eq. (9) is the polynomial chaos expansion [31]. This equation is called surrogate model or meta-model. In this study, the closed-form expressions for fourth to sixth order Hermit polynomial chaos expansions involving any number of random variables, formulated by Li et al. [17], are used.

3. Estimation of the unknown coefficients in polynomial chaos expansion. For this purpose, the collocation point method [15] is used. The model outputs are computed at a set of collocation points and used to estimate the unknown coefficients. Collocation points must be selected from all combinations of roots of the polynomial of one degree higher than the order of the polynomial chaos expansion $[15,32,33]$. For the second-order chaos polynomials, the roots of the third-order chaos polynomials are 0 and $\pm \sqrt{3}$. For $N$-dimensional and $p$-order chaos polynomials $(p+1)^{N}$, combinations of the roots exist which are always larger than the number of the collocation points needed. For example, for third-order and eight-dimensional chaos polynomials, the number of available collocation points is $(3+1)^{8}=65536$ while $\frac{(8+3) !}{8 ! 3 !}=165$ points are needed.

Selection of the appropriate collocation points from the large number of potential candidates is a practical question. There are some criteria for the selection of collocation point in the literature $[13,15,18,32]$. Selected points must capture regions of high probability. The points, which are closer to the origin of the multivariate normal space, are preferred. It is desirable to achieve a symmetric distribution of collocation points with respect to the origin because the Probability Density Function (PDF) is symmetric with respect to the origin.

When $n$ sets of collocation points are selected, the corresponding model outputs $\mathbf{y}=$ $\left[y_{1}, y_{2}, \ldots, y_{n}\right]^{T}$ at each set of collocation points can 


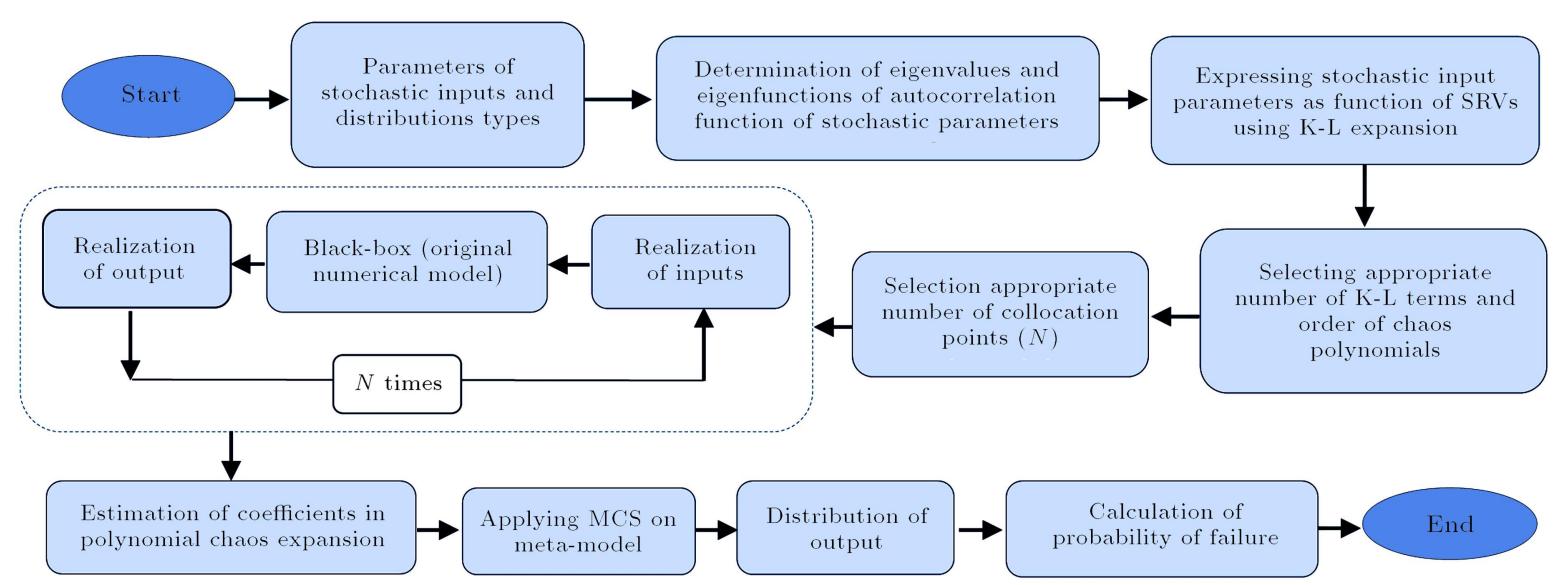

Figure 1. Flowchart of SRSM.

be obtained. The unknown coefficients can be computed using the following equation:

$$
\mathbf{C}=\left(\mathbf{Z}^{\mathbf{T}} \mathbf{Z}\right)^{-1} \cdot \mathbf{Z}^{\mathbf{T}} \cdot \mathbf{y}
$$

where $\mathbf{C}=\left[c_{1}, c_{2}, \ldots, c_{P}\right]$ is a vector of unknown coefficients, and $\mathbf{Z}$ (Hermit polynomial matrix) is a space-independent matrix of dimension $N \times P$ and is given as follows:

$$
\mathbf{Z}=\left[\begin{array}{ccccc}
\psi_{0}^{1}(\boldsymbol{\xi}) & \psi_{1}^{1}(\boldsymbol{\xi}) & \psi_{2}^{1}(\boldsymbol{\xi}) & \ldots & \psi_{P-1}^{1}(\boldsymbol{\xi}) \\
\psi_{0}^{1}(\boldsymbol{\xi}) & \psi_{1}^{1}(\boldsymbol{\xi}) & \psi_{2}^{1}(\boldsymbol{\xi}) & \ldots & \psi_{P-1}^{1}(\boldsymbol{\xi}) \\
\vdots & \vdots & \vdots & & \vdots \\
\vdots & \vdots & \vdots & & \vdots \\
\psi_{0}^{N}(\boldsymbol{\xi}) & \psi_{1}^{N}(\boldsymbol{\xi}) & \psi_{2}^{N}(\boldsymbol{\xi}) & \ldots & \psi_{P-1}^{N}(\boldsymbol{\xi})
\end{array}\right](13)
$$

It should be mentioned that the number of collocation points selected should ensure that $\mathbf{Z}^{\mathbf{T}} . \mathbf{Z}$ is invertible. Flowchart of the SRSM is presented in Figure 1.

\section{Random finite-element method}

The RFEM is one of the most accurate, yet time expensive, methods to analyze reliability of finiteelement models. In this method, a random field of soil properties is generated and then mapped onto a finiteelement mesh [7]. The steps of this method using K-L expansion are as follows:

1. Representing the statistical properties of stochastic input variables, such as mean, standard deviation, autocorrelation function, and distribution type;

2. Generating random field using K-L expansion;

3. Performing finite-element analysis using generated random field to compute system response;

4. Repeating steps 2 and 3 many times using MCS to obtain the histogram of system response;

5. Computing the statistical properties of system response such as mean, standard deviation, and probability of failure $\left(P_{f}\right)$.

The flowchart of this method is shown in Figure 2.

\section{Computer program}

In this study, for reliability analysis of foundation settlement, two finite-element programs were coded by MATLAB based on SRSM, RFEM. These programs are capable of considering the uncertainties of soil

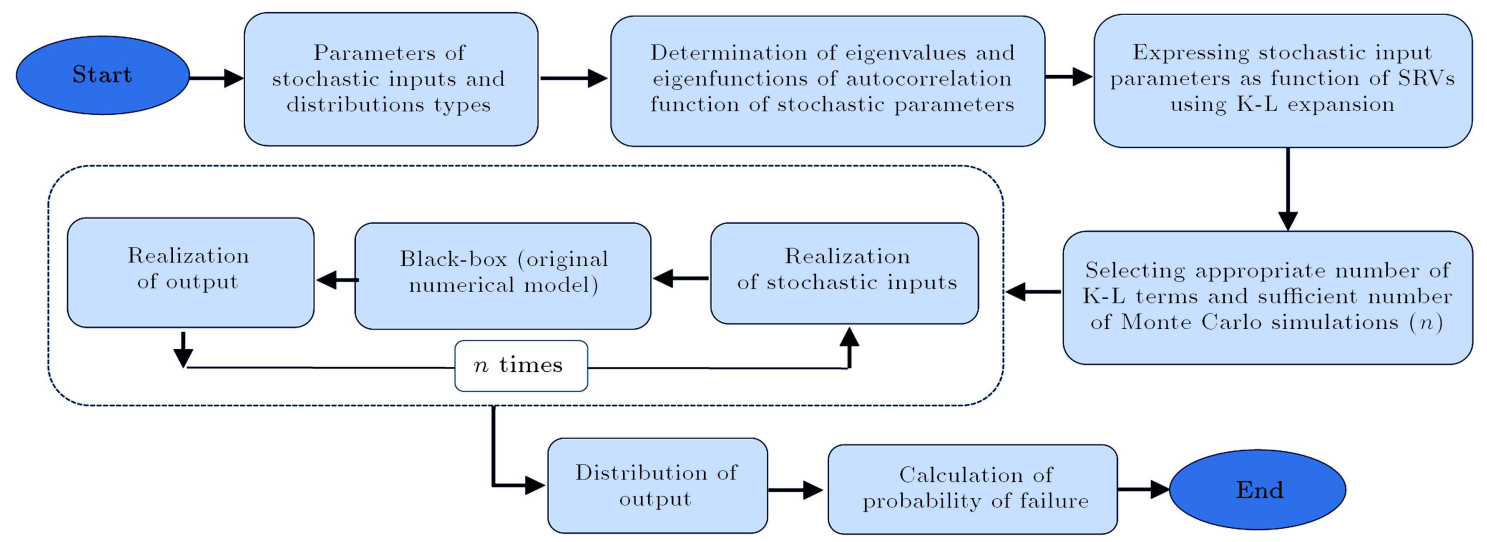

Figure 2. Flowchart of RFEM. 
parameters. The major capabilities of these programs are as follows:

1. Considering elastic-perfectly plastic behavior of the soil material with Mohr-Coulomb yield criterion;

2. Selecting appropriate collocation points automatically (SRSM program);

3. Generating random field by K-L expansion considering cross correlation between stochastic parameters;

4. Computing chaos polynomials for any number of random variables based on closed-form expressions (SRSM program).

\section{Verification of the developed programs}

To verify the accuracy of the developed programs, a deterministic analysis was done using mean values of soil parameters, given in Table 1. The obtained result, including the foundation pressure-settlement curve, is compared with the prediction result of finite-element software, PLAXIS. For this purpose, soil mass with $13 \mathrm{~m}$ width and the depth of $7 \mathrm{~m}$ is discretized into 52 four-node rectangular elements in the horizontal direction by 5 elements in vertical direction, as shown in Figure 3. The nodes on the bottom boundary are fixed and both lateral boundaries are assumed roller. A strip footing with a width of $B=3.0 \mathrm{~m}$ is located on a $c-\phi$ soil layer. A uniform footing pressure equal to $150 \mathrm{kPa}$ is applied onto the top of soil layer.

The ultimate settlements at the center of footing

Table 1. Arbitrary selected soil parameters for modelling.

\begin{tabular}{lc}
\hline Parameter & Value \\
\hline Young's modulus $E(\mathrm{MPa})$ & 30 \\
Poisson's ratio $v$ & 0.3 \\
Cohesion $c(\mathrm{kPa})$ & 20 \\
Friction angle $\phi($ deg. $)$ & 25 \\
Dilation angle $\alpha($ deg. $)$ & 0 \\
Unit weight $\gamma\left(\mathrm{kN} / \mathrm{m}^{3}\right)$ & 0 \\
\hline
\end{tabular}

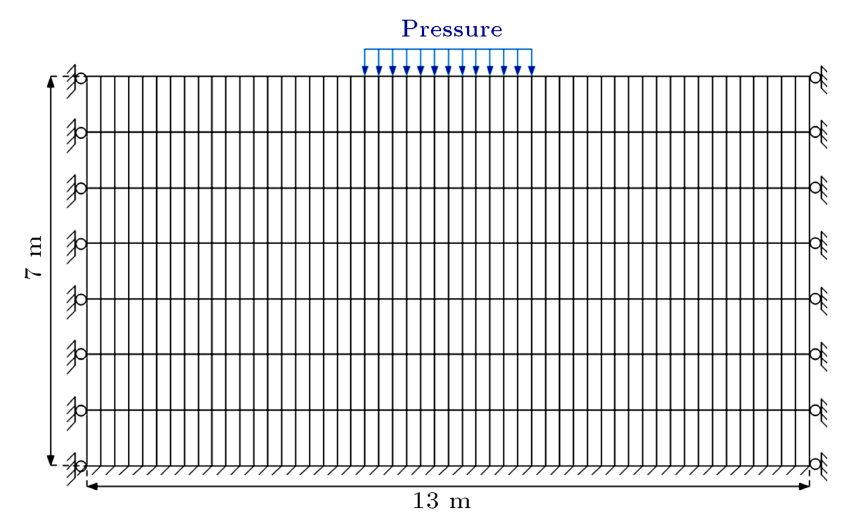

Figure 3. Finite element model of soil mass.

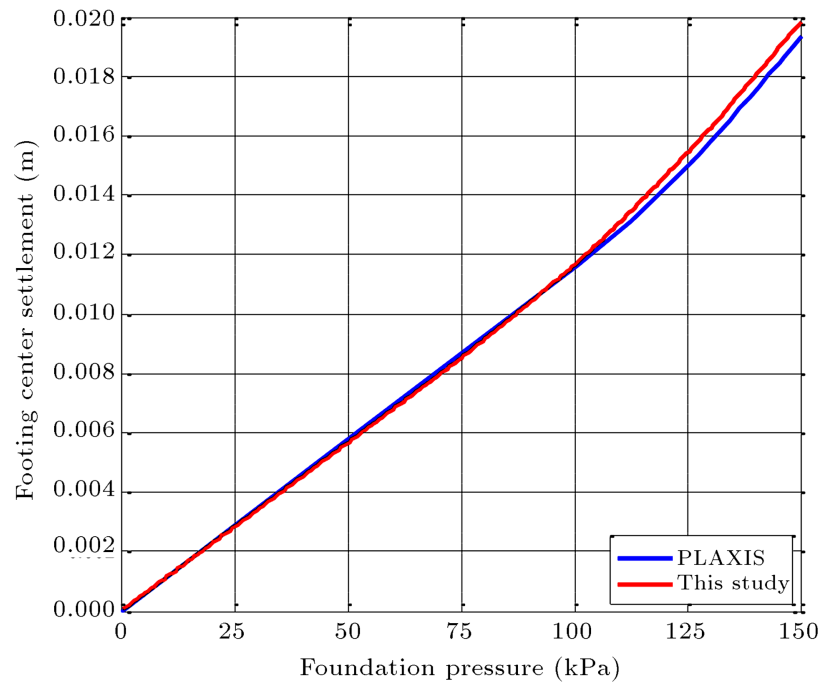

Figure 4. The foundation pressure-settlement curves obtained using developed program and PLAXIS.

(Sc) due to the applied pressure using the developed program and PLAXIS were obtained as $0.0198 \mathrm{~m}$ and $0.0193 \mathrm{~m}$, respectively. The results show that the developed program can predict settlement with reasonable accuracy. The foundation pressure-settlement curves obtained using the developed program and PLAXIS are shown in Figure 4. This figure shows that the results are close to each other, and the developed program successfully predicted the foundation settlement.

\section{Illustrative example}

To compare the proposed methods in reliability analysis of foundation settlement with others, an illustrative example with arbitrary data is presented. In this example, the elastic-perfectly plastic Mohr-Coulomb yield criterion is adopted to represent the stress-strain behavior of the soil. The mean of soil parameters is selected as in Table 1, and the geometry of problem is considered as in Figure 3. The values of soil parameters are selected arbitrarily.

Only soil elastic properties $E$ and $v$ are considered as uncertain parameters $[6,7,27,34]$ and modeled as cross correlated lognormal random fields. There is no sufficient information about coefficient of variation of Poisson's ratio. Some authors have suggested that the variability of this parameter can be neglected, while others proposed a very limited range of variability [11]. In this research, Coefficient Of Variation (COV) of $v$ is selected equal to 0.05 as Youssef Abdel Massih [35] and $\mathrm{COV}$ of $E$ is selected equal to 0.3 . A value of -0.5 is used for $R_{E, v}$ [35].

The K-L expansion is employed to discretize random field. The type of autocorrelation functions of uncertain parameters can be different from each other, but in this example, it is assumed that both 


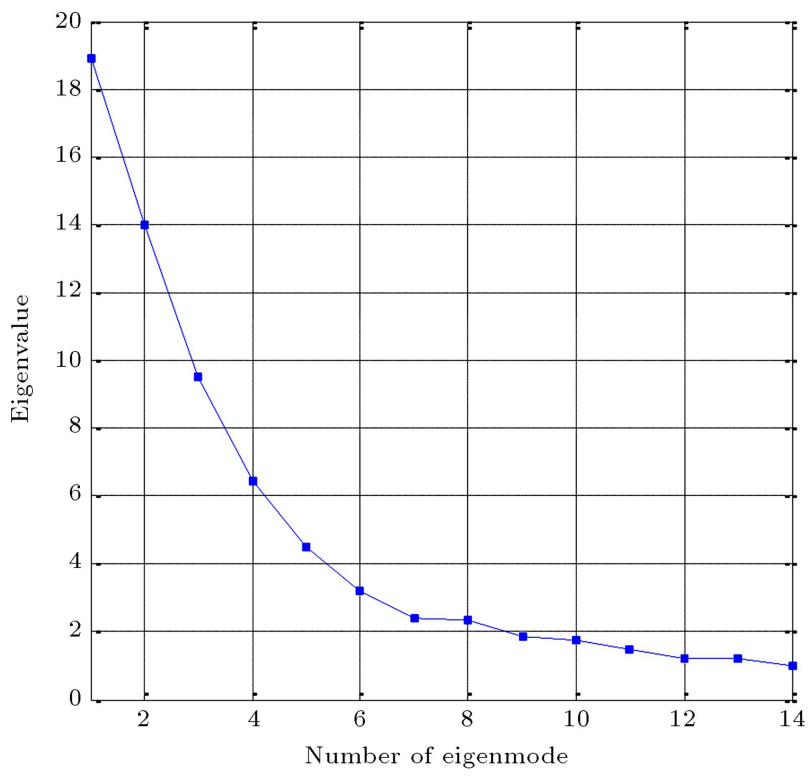

Figure 5. Eigenvalues of 2-D autocorrelation function.

stochastic parameters have the same autocorrelation function as in Eq. (1). It should be emphasized here that the autocorrelation function and autocorrelation length are generally site-specific and often challenging due to insufficient site data and high cost of site investigations [11]. Random field is calculated at the centroid of each element. The first 14 eigenvalues of $2 \mathrm{D}$ autocorrelation function, where values of $l_{x}=$ $20 \mathrm{~m}$ and $l_{y}=1 \mathrm{~m}$ are considered for horizontal and vertical autocorrelation lengths, respectively, are shown in Figure 5. In this example, in all cases, 6 terms of K-L expansion are considered first.

Analyzed by SRSM, polynomials chaos of order 2 is adopted. As nonlinearity of problem is increased, the greater order of polynomials chaos must be selected. Eigenvalues and eigenfunctions of autocorrelation function are computed, and the first 6 terms of K-L expansion (the first 6 Eigenvalues and eigenfunctions of autocorrelation function) of each spatial random variable $(E$ and $v)$ are considered. Number of unknown coefficient, which must be evaluated, is 91 ; therefore, 91 collocation points are needed. 106 collocation points are selected, greater than the number of the needed collocation points for the robust estimation of unknown coefficients. After computing the unknown coefficients, MCS with 100,000 sampling is applied to meta-model (Eq. (9)). The histogram and fitted PDF of center of foundation settlement (Sc) determined using SRSM are shown in Figure 6.

In stochastic analysis, using RFEM, eigenvalues and eigenfunctions of autocorrelation function are computed first, and like SRSM, the first 6 terms of K$\mathrm{L}$ expansion are considered. The random field has been generated 10,000 times, and the results are used in original finite-element model to determine the Sc.

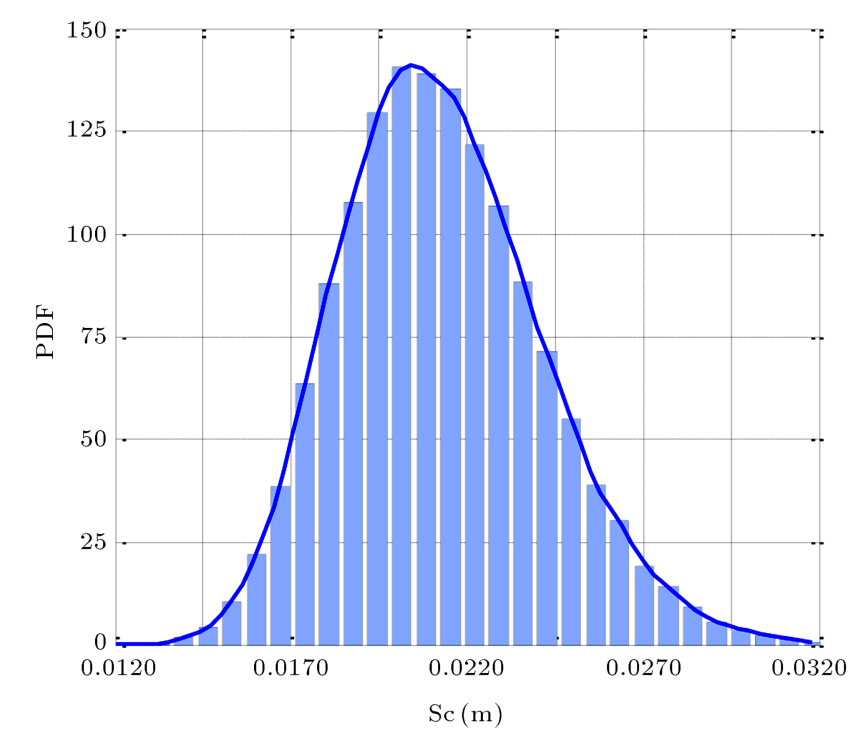

Figure 6. The histogram and PDF of Sc obtained using SRSM.

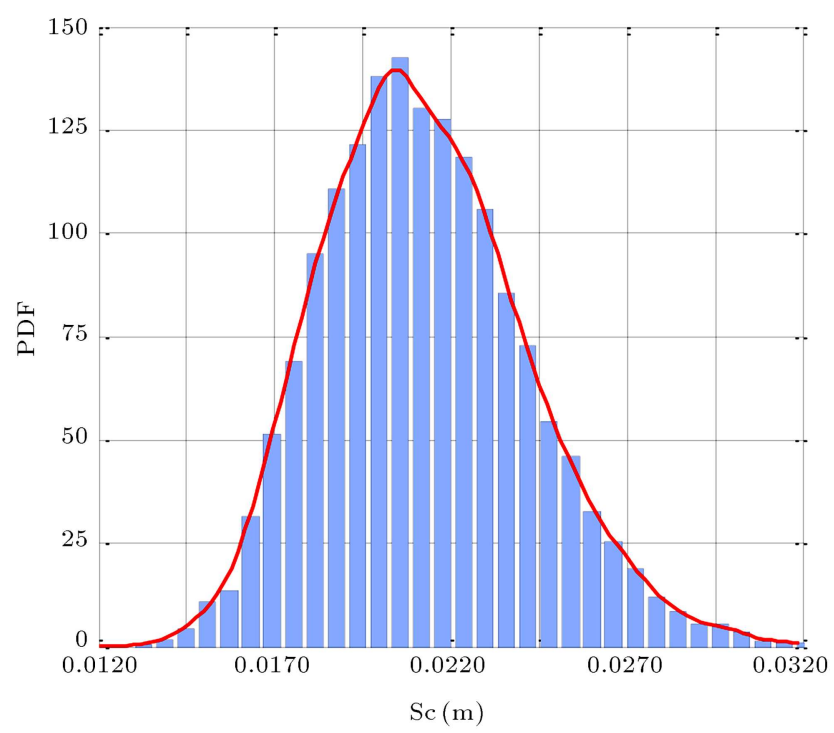

Figure 7. The histogram and PDF of Sc obtained using RFEM.

The histogram and fitted PDF of the Sc determined using RFEM are shown in Figure 7. For comparing the determined PDF and Cumulative Distribution Function (CDF) by two methods, they are plotted in one figure. Figures 8 and 9 show the determined PDF and CDF of two methods, respectively. These figures show that the results of two methods are close to each other; however, the computational time of the SRSM is considerably less than RFEM.

By assuming the threshold value of footing center settlement equal to $0.025 \mathrm{~m}$ and the required time of two methods, the probability of system failure $\left(P_{f}\right)$ is given in Table 2. It can be seen that the results of SRSM are close to those of RFEM; however, the 


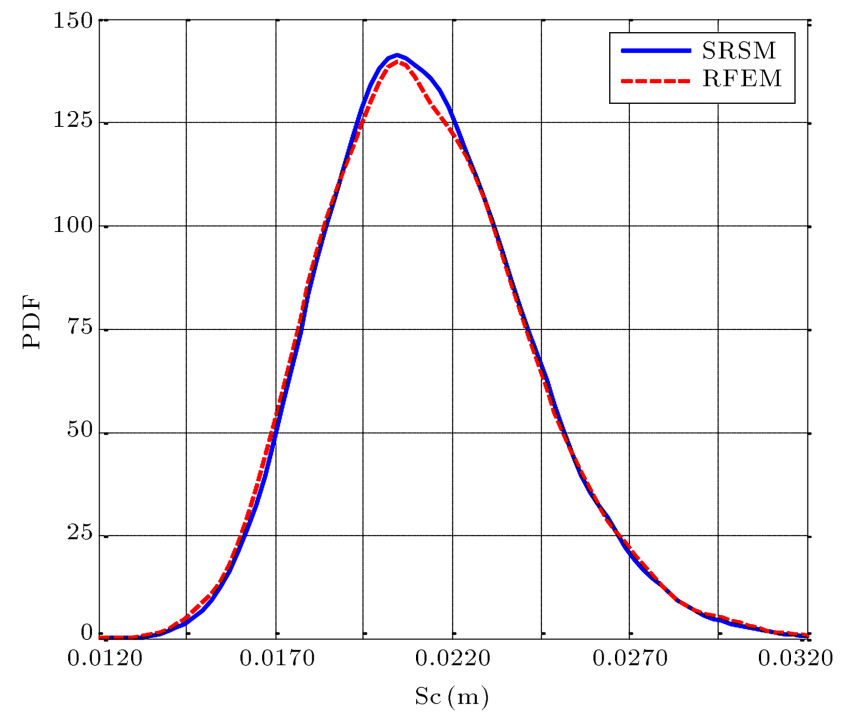

Figure 8. The PDFs of Sc obtained using SRSM and RFEM.

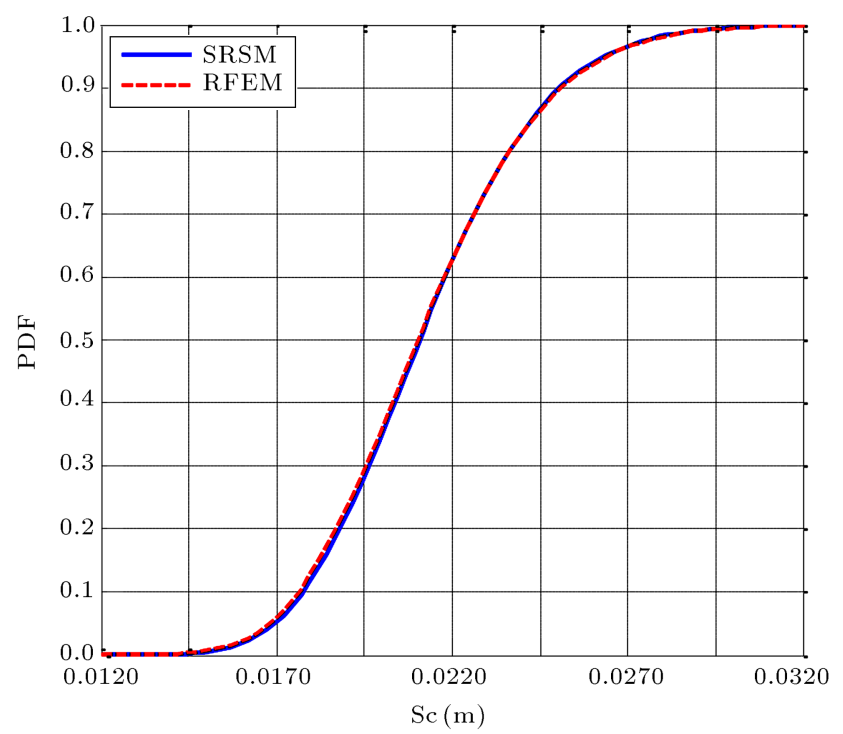

Figure 9. The CDFs of Sc obtained using SRSM and RFEM.

consumed time in RFEM is at most 50 times longer than that of SRSM.

In this study, the effect of autocorrelation length on PDF of system is investigated. For this purpose, the SRSM with less computational time is used. The PDFs of system response for different values of autocorrelation length and homogenous soil layer $\left(l_{x}=\infty, l_{y}=\infty\right)$ are shown in Figure 10.

This figure shows that as the autocorrelation

Table 2. Comparison of results of SRSM and RFEM.

\begin{tabular}{ccccc}
\hline Method & $\boldsymbol{\mu}_{S c}$ & $\boldsymbol{\sigma}_{S \boldsymbol{c}}$ & $\boldsymbol{P}_{\boldsymbol{f}}(\boldsymbol{\%})$ & Time (hour) \\
\hline SRSM & 0.0213 & 0.0029 & 10.29 & 6.80 \\
RFEM & 0.0212 & 0.0029 & 10.04 & 360.5 \\
\hline
\end{tabular}

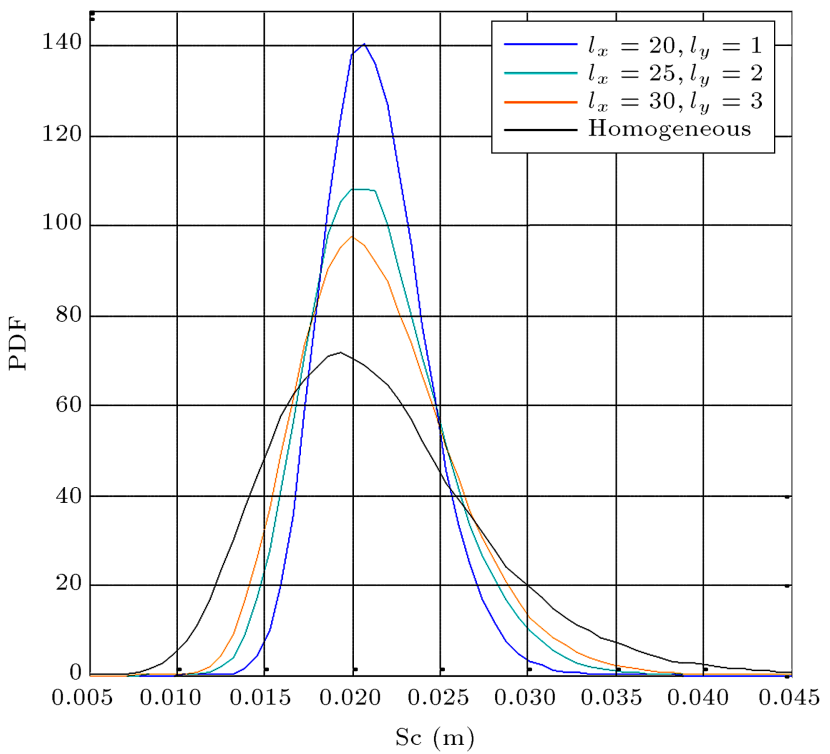

Figure 10. The PDFs of Sc for different values of autocorrelation lengths obtained by SRSM.

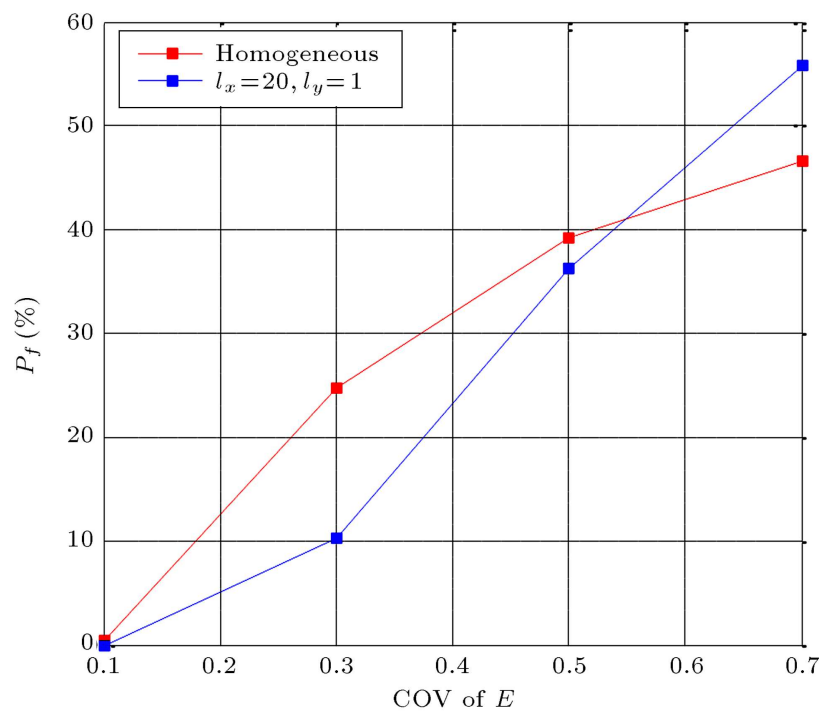

Figure 11. $P_{f}$ for various COVs of $E$ by assuming the threshold value of Sc equal to $0.025 \mathrm{~m}$.

lengths increase, the system response dispersion increases too and the case of homogenous soil has maximum dispersion. In this analysis, COVs of $v$ and $E$ are selected equal to 0.05 and 0.30 , respectively.

The COV and $P_{f}$ of Sc for different values of autocorrelation lengths are presented in Table 3. From this table, it can be seen that in this example, both $P_{f}$ and COV increase with an increase in autocorrelation lengths, and $P_{f}$ and $\mathrm{COV}$ show the largest values for homogenous soil.

Figures 11 and 12 summarize the variations of the probability of failure with the coefficient of variation of $E$ for both cases of ignoring and considering spatial variation of stochastic parameters. These figures show 
Table 3. $P_{f}$ and COV of Sc for different values of autocorrelation lengths.

\begin{tabular}{|c|c|c|c|c|}
\hline Parameter & $\begin{array}{c}l_{x}=20 \mathrm{~m} \\
l_{y}=1 \mathrm{~m}\end{array}$ & $\begin{array}{c}l_{x}=25 \mathrm{~m} \\
l_{y}=2 \mathrm{~m}\end{array}$ & $\begin{array}{c}l_{x}=30 \mathrm{~m} \\
l_{y}=3 \mathrm{~m}\end{array}$ & Homogenous \\
\hline$P_{f}(\%)$ & 10.29 & 16.18 & 19.07 & 24.7 \\
\hline $\mathrm{COV}$ & 0.1345 & 0.1747 & 0.1986 & 0.2837 \\
\hline
\end{tabular}

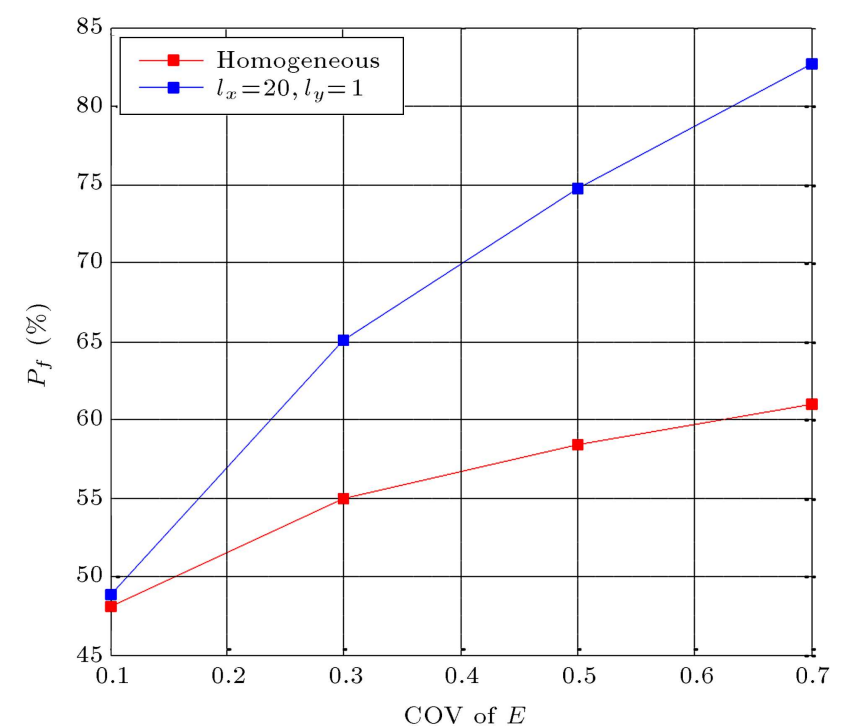

Figure 12. $P_{f}$ for various COVs of $E$ by assuming the threshold value of Sc equal to $0.02 \mathrm{~m}$.

the results of two values of threshold footing center settlement: $0.025 \mathrm{~m}$ (Figure 11) and $0.020 \mathrm{~m}$ (Figure 12).

This figure shows that the probability of failure increases with increasing COV of $E$. Figure 11 shows that for COVs of $E$ smaller than 0.7, the predicted $P_{f}$ of homogenous soil is greater than when spatial variability is considered, whereas Figure 12 does not show these results. Based on these figures, it can be concluded that to have a correct result in stochastic analysis, it is necessary to consider the spatial variability of soil properties.

\section{Conclusions}

In this study, the reliability analysis of foundation settlement by SRSM and RFEM was presented. For this purpose, two finite-element programs were coded by MATLAB based on two methods. The elastic soil properties were considered as spatial random variables and modeled as cross correlated log normal random field. The results of two methods were compared with each other, and it is observed that the results of two methods are close to each other. In the presented example, to reach the same result, in RFEM, the deterministic numerical model must be run 104 times, while SRSM model must be run 106 times. It is concluded that the SRSM is more time efficient compared with RFEM, because in SRSM, MCS is applied onto meta-model, whereas in RFEM, the MCS is applied onto the original numerical model, which is more time-consuming compared with meta-model. The effect of autocorrelation length was investigated by SRSM. It was shown that in stochastic analysis, to have a correct result, it is necessary to consider the spatial variability of soil properties.

The approximate real soil behavior, which includes hardening or softening, has not been considered in this research. The authors suggest the following future studies for further improvements on and extension to the topic:

- Three-dimension analysis of foundation settlement by SRSM.

- Stochastic analysis of differential settlement.

- Stochastic analysis of settlement problems with seismic or dynamic loading.

\section{References}

1. Chau, K.W. "Reliability and performance-based design by artificial neural network", Adva. Eng. Soft., 38, pp. 145-149 (2007).

2. Cheng, C.T., Li, X.Y. and Chau, K. "A parallel adaptive metropolis algorithm for uncertainty assessment of Xinanjiang model parameters", IAHS-AISH publi., pp. $10-16$ (2008).

3. Maheshwari, P. "Settlement of shallow footings on layered soil: State-of-the-art", Int. J. Geot. Eng., 9, pp. $42-48$ (2015).

4. Dodagoudar, G. and Shyamala, B. "Finite element reliability analysis of shallow foundation settlements", Int. J. of Geo. Eng., 9(3), pp. 316-26 (2015).

5. Fenton, G.A. and Griffiths, D. "Statistics of block conductivity through a simple bounded stochastic medium", Wate. Resou. Res., 29, pp. 1825-1830 (1993).

6. Fenton, G.A. and Griffiths, D. "Probabilistic foundation settlement on spatially random soil", J. of Geotech. and Geoen. Eng., 128(5), pp. 381-390 (2002).

7. Griffiths, D. and Fenton, G.A. "Probabilistic settlement analysis by stochastic and random finite-element methods", J. Geot. Geoen. Eng., 135, pp. 1629-1637 (2009). 
8. Johari, A., Rezvani Pour, J. and Javadi, A. "Reliability analysis of static liquefaction of loose sand using the random finite element method", Eng. Comp., 32(7), pp. 2100-2119 (2015).

9. Paice, G., Griffiths, D. and Fenton, G.A. "Finite element modeling of settlements on spatially random soil", J. Geot. Eng., 122(9), pp. 777-779 (1996).

10. Pieczynska-Kozlowska, J., Pula, W. Griffiths, D. and Fenton, G. "Influence of embedment, self-weight and anisotropy on bearing capacity reliability using the random finite element method", Comp. Geot., 67, pp. 229-238 (2015).

11. Ahmed, A. "Simplified and advanced approaches for the probabilistic analysis of shallow foundations", Nantes (2012).

12. Berveiller, M., Sudret, B. and Lemaire, M. "Stochastic finite element: a non intrusive approach by regression", Eur. J. Comp. Mech., 15, pp. 81-92 (2006).

13. Huang, S., Liang, B. and Phoon, K. "Geotechnical probabilistic analysis by collocation-based stochastic response surface method: An Excel add-in implementation", Geori., 3(2), pp. 75-86 (2009).

14. Huang, S., Mahadevan, S. and Rebba, R. "Collocationbased stochastic finite element analysis for random field problems", Prob. Eng. Mech., 22(2), pp. 194-205 (2007).

15. Isukapalli, S., Roy, A. and Georgopoulos, P. "Stochastic response surface methods (SRSMs) for uncertainty propagation: application to environmental and biological systems", Ris. anal., 18(3), pp. 351-363 (1998).

16. Jiang, S.H., Li, D.Q., Zhang, L.M. and Zhou, CB. "Slope reliability analysis considering spatially variable shear strength parameters using a non-intrusive stochastic finite element method", Eng. geol., 168, pp. 120-128 (2014).

17. Li, D., Chen, Y., Lu, W. and Zhou, C. "Stochastic response surface method for reliability analysis of rock slopes involving correlated non-normal variables", Comp. Geot., 38(1), pp. 58-68 (2011).

18. Li, H. and Zhang, D. "Probabilistic collocation method for flow in porous media: Comparisons with other stochastic methods", Wat. Reso. Rese., 43(W09409), pp. $44-48$ (2007).

19. Soubra, A.H. and Mao, N. "Probabilistic analysis of obliquely loaded strip foundations", Soi. foun., 52(3), pp. 524-538 (2012).

20. El-Ramly, H., Morgenstern, N. and Cruden, D. "Probabilistic slope stability analysis for practice", Can. Geot. J., 39(3), pp. 665-683 (2002).

21. Lacasse, S. and Nadim, F. "Uncertainties in characterising soil properties", Publ.-Norg. Geot. Inst., 201, pp. 49-75 (1997).
22. Vanmarcke, E., Random Fields: Analysis and Synthesis, MIT Press, Cambridge, MA (1983).

23. Baecher, G.B. and Christian, J.T., Reliability and Statistics in Geotechnical Engineering, John Wiley \& Sons (2005).

24. Sudret, B. and Der Kiureghian, A. "Stochastic finite element methods and reliability: a state-of-the-art report", Berkeley, CA: Dep. Civ. Env. Eng., Uni. Cali. (2000).

25. Ghiocel, D.M. and Ghanem, R.G. "Stochastic finiteelement analysis of seismic soil-structure interaction", J. Eng. Mech., 128(1), pp. 66-77 (2002).

26. Ghanem, R.G. and Spanos, P.D. "Spectral Stochastic Finite-Element Formulation for Reliability Analysis", J. Eng. Mech., 117(10), pp. 2351-2372 (1991).

27. Ghanem, R.G. and Spanos, P.D., Stochastic Finite Elements: A Spectral Approach, Courier Corporation (2003).

28. Zhang, D. and Lu, Z. "An efficient, high-order perturbation approach for flow in random porous media via Karhunen-Loeve and polynomial expansions", $J$. Comp. Phys., 194(2), pp. 773-794 (2004).

29. Cho, S.E. and Park, H.C. "Effect of spatial variability of cross-correlated soil properties on bearing capacity of strip footing", Int. J. Num. Anal. Meth. Geom., 34(1), pp. 1-26 (2010).

30. Vov̌echovský, M. "Simulation of simply cross correlated random fields by series expansion methods", Struc. Saf., 30(4), pp. 337-363 (2008).

31. Sudret, B. "Polynomials chaos expansions and stochastic finite element methods", Ris. Reli. Geot. Eng., (Chapter 6), pp. 265-300 (2015).

32. Tatang, M.A., Pan, W., Prinn, R.G. and McRae, G.J. "An efficient method for parametric uncertainty analysis of numerical geophysical models", J. Geoph. Rese.: Atmospheres (1984-2012), 102, pp. 2192521932 (1997).

33. Webster, M.D. Tatang, M.A. and McRae, G.J. "Application of the probabilistic collocation method for an uncertainty analysis of a simple ocean model" , MIT joint program on the science and policy of global change report series no. 4, Massachusetts Institute of Technology (1996).

34. Babu, G.S. and Srivastava, A. "Reliability analysis of allowable pressure on shallow foundation using response surface method", Comp. Geot, 34(3), pp. 187-194 (2007).

35. Youssef Abdel Massih, D.S. and Soubra, A.H. "Reliability-based analysis of strip footings using response surface methodology", Int. J. Geom., 8(2), pp. 134-143 (2008).

\section{Biographies}

Ali Johari obtained his $\mathrm{BS}, \mathrm{MS}$, and $\mathrm{PhD}$ degrees in 1995, 1999, and 2006, respectively, from Shiraz 
University, Iran, where he is currently an Assistant Professor in the Civil and Environmental Engineering Department. He was a Post-Doctoral Researcher at Exeter University in 2008, where he is also a member of the research staff of the Computational Geomechanics Group. His research interests include unsaturated soil mechanics, application of intelligent systems in geotechnical engineering, probabilistic models and reliability assessment. He has also consulted and super- vised numerous geotechnical projects.

Abouzar Sabzi was born in Abdanan, Iran, in 1990. He received his BS degree in Civil Engineering from the Ilam University, Ilam, Iran, in 2012. He is studying Geotechnical Engineering at Shiraz University of Technology to obtain MS degree. His current main interests of research are numerical methods and stochastic analysis in geotechnical engineering. 